\title{
Research on Haze Characteristics in Chongqing Jiangbei Airport from 2001 to 2016
}

\author{
Xiaoning Zhou ${ }^{1}$, Qian Geng ${ }^{1, *}$, Xingyu $\mathrm{Li}^{2}$, Dong $\mathrm{Chen}^{3}$ \\ ${ }^{1}$ Nanhang Jincheng College, Nanjing, Jiangsu, China \\ ${ }^{2}$ LACS, Institute of Atmospheric Physics, CAS, Beijing, China \\ ${ }^{3}$ PLA 95871 troops, Hengyang, Hunan, China \\ *Corresponding author
}

Keywords: Chongqing, Haze, Meteorological conditions, Characteristics.

\begin{abstract}
In the present study, a comprehensive analysis of the characteristics of haze and meteorological conditions in areas of Chongqing Jiangbei Airport(ZUCK) were conducted using hourly surface observation data in the airport for the period 2001-2016. It was found that the appearing frequency of haze and severe hazes was the most in winter, while the lowest in July. In addition, events occurred frequently from 11:00 to 21:00 and infrequently in the early morning from 00:00 to 07:00. The highest frequency of haze and severe haze was often mixed with the north wind, and the average wind speed was usually $1-2 \mathrm{~m} \mathrm{~s}^{-1}$. Events occurred infrequently when the wind speed was more than $4 \mathrm{~m} \mathrm{~s}^{-1}$. The relative humidity between $50 \%$ and $70 \%$ was found to be throughout haze and severe haze events. Most events were mixed with mist, whereas not mixed with other weather phenomena.
\end{abstract}

\section{Introduction}

Haze is traditionally an atmospheric phenomenon where dust, smoke and other dry particles obscure the clarity of the sky, making horizontal visibility less than $10 \mathrm{~km}^{1}$. It is also known as dust-haze. The haze can reduce visibility, increase the occurrence probability of traffic accidents, lead to flight delay and even flight cancellation. Haze not only affects the traffic safety, atmospheric environmental quality, city images and the physical and mental health, it will also affect the regional climate, leading to frequent extreme weather events.

Chongqing, located in the eastern part of the Sichuan basin, is a key area of haze control and one of the key control areas of dust-haze. In recent years, with the rapid development of the economy and the acceleration of urbanization in Chongqing, the rapid growth of urban energy consumption leads to the increase of pollutant emissions. And the geographic meteorological conditions in Chongqing are not conducive to the spread of pollutants, the haze pollution here has aroused a growing concern.

The weather in downtown Chongqing is dominated by haze, and the intensity of haze is mainly slight ${ }^{2}$. According to the research of Yihui Ding and Yanju Liu, the haze has had a tendency of declining in the central and western regions for the past 50 years. Between 1997 and 2012, although there was no significant change in the number of days of haze, the severity of the haze was reduced ${ }^{2,4}$. In 2011, pollution levels of haze in the Chongqing area were gradually shifted from severe to light/medium, and the condition was getting better ${ }^{5}$. The haze in Chongqing is mainly in autumn and winter, and it is an important part of atmospheric particulates4. The number of haze days in winter is the most and lasts longer, while the number of haze days in summer is the least and the level is the lightest ${ }^{2}$. At the same time, the lower wind speed (speed $\leqslant 2.0 \mathrm{~m} / \mathrm{s}$ ), and higher relative humidity is good for the haze ${ }^{6}$.

As can be seen from the existing research, the analysis of haze characteristics is based on the daily meteorological data of meteorological offices and stations. It is impossible to analyze the characteristics such as hourly change of haze, the wind direction/wind speed and the accompanied 
weather. To further understand the characteristics of haze, based on the hourly surface observation data in Chongqing Jiangbei Airport, this paper does more elaborate analysis on characteristics of inter-annual variation, time variation and meteorological conditions of the airport. It is of great significance to provide correct understanding of haze weather, improve the accuracy of the forecast, ensure aviation security and provide scientific basis for atmospheric monitoring and controlling.

\section{Data and Methods}

Chongqing Jiangbei International Airport is located in northeast of Chongqing, Yubei District. It is one of the eight major hub airports in China. The hourly surface observation data is from National Climatic Data Center, NCDC. The observation data in the airport is recorded by the observation instruments and facilities stipulated by relevant observation specification8 and Industry Standards of Civil Aviation in the People's Republic of China MH/T 4016 $6^{8,9}$. It is also reported and stored according to relevant specifications ${ }^{8-9}$.

In the early days, there was no accurate understanding of fog and haze. There was no uniform national standard for haze, which was often recorded as light fog or fog ${ }^{7}$. If haze was judged by surface observation (or other information), then it would probably be a misjudgment.

Before 2001, the surface observation data in the airport was made every three hours. From 2001 to 2016, the airport observation data was made every hour. The time resolution was relatively high, and the haze observation was relatively accurate. Thus, the observation data of this time period is chosen to analyze the characteristics of haze.

If there is haze in a weather phenomenon record in the routine observation at a certain time level, then it will be defined as a haze observation record. According to the Observation and Forecasting Levels of Haze (Dust-Haze) (QX/T113-2010)released by China Meteorological Administration, Visibility less than $2 \mathrm{~km}$ is defined as severe haze ${ }^{1}$. In the end, there were 6,687 records of haze and 654 records of severe haze.

When haze occurs and the temperature is generally higher than $-40^{\circ} \mathrm{C}$, the relative humidity can be obtained by the following formula ${ }^{10}$ :

$$
\left\{\begin{array}{l}
E_{S}=1.000071 e^{0.0000045 p} E \\
E=6.1094 e^{\frac{17.625 T}{243.04+T}} \\
R H=\frac{E_{S}\left(T_{d}\right)}{E_{S}(T)}
\end{array}\right.
$$

In this formula, , $\mathrm{P}$ is the pressure (hPa), $\mathrm{T}$ is the temperature $\left({ }^{\circ} \mathrm{C}\right), \mathrm{E}$ is the vapor pressure $(\mathrm{hPa})$, Es is the saturated vapor pressure (hPa), $\mathrm{RH}$ is the relative humidity (\%).

At present, the number of days is often used to study the intensity of haze ${ }^{2-6}$. Since hourly observation data is used in this paper, the frequency and strength of haze are characterized by the time level and the accumulative annual hours ${ }^{11-12}$.

In the analysis of the characteristics of meteorological conditions in the occurrence of haze, the records of the missing measurement of corresponding meteorological elements are not calculated. In order to avoid the effect of individual extreme events on the statistical results, reduce the result errors, and make the result more statistically significant, the analysis adopts index such as average and weighted average.

When analyzing the time distribution of haze weather, the coordinated universal time (UTC) recorded in the civil aviation observation records is switched into Beijing time, and the data is smoothed by cubical smoothing algorithm with five-point approximation. 


\section{Result Analysis}

The Characteristics of Inter-Annual Variety. The annual hours distribution graph of haze in Chongqing Jiangbei Airport from 2001 to 2016 (shown in Figure 1) is based on the annual accumulated total of haze hours. Over the past 16 years, the annual hours of haze has been above 300h. Between 2001 and 2005, the overall trend was upward. 2005 - 2009 showed a downward trend. 2009 - 2013 showed a upward trend. 2013 - 2016 showed a downward trend. From the haze hours in the past years, it is easy to see that The number of haze hours was higher in 2006, 2011 and 2013, the highest number of severe haze hours was in 2011. In the analysis of Wei Huang and other people4, days of regional haze in Chongqing increased more in 2011 than in 2010. The results is basically consistent with the paper. The annual haze hours is around $40 \mathrm{~h}$ in the rest years.

In Wenhua Jiang's analysis with other people ${ }^{4}$ of variety tendency of haze days in main urban area in Chongqing, it is found that in 2000-2006, it was almost on the rise, 2007-2012 was down. There was a difference between the main urban area of Chongqing and the annual variety characteristics of Jiangbei Airport. However, in 2006, Chongqing was extremely hot and dry, with less rainfall and more haze. The haze hours were more in Jiangbei Airport too. There was certain synchronicity between the two areas in 2006.

Since the "Blue Sky" was put into effect in urban area of Chongqing in 2005, the haze and severe haze of the Jiangbei Airport have declined in 2005-2009. But with the development of the urban area in Chongqing, Jiangbei Airport also has a slightly different character from the main city. It is suggested that haze has certain local characteristics, which need to be analyzed specifically according to specific areas.

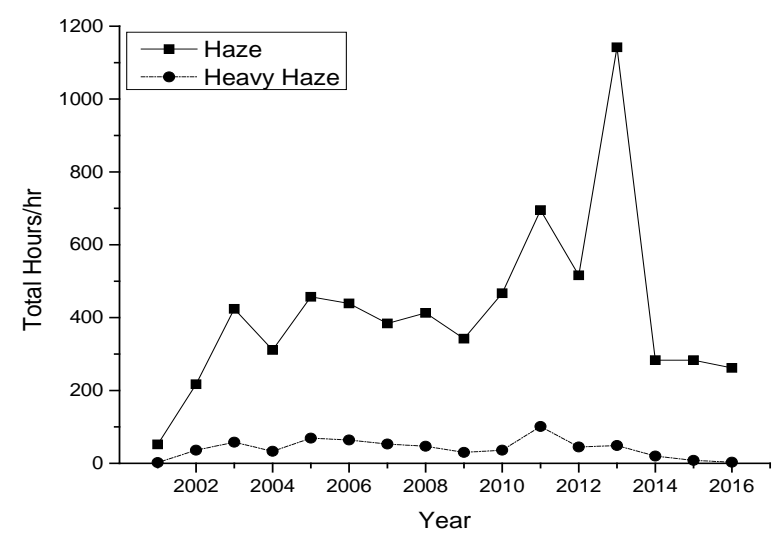

Figure 1. Distribution Graph Of Annual Haze Hours And Severe Haze Hours From 2001 To 2006

The Variety Characteristics of Time. From the distribution of haze in each month (shown in Figure 2), it is easy to see that the haze was most likely to occur in December, January and February, and the haze in February took the largest proportion (19.23\%), 1286 records in total, and then gradually decreased. There was less haze in summer. In July the proportion was the smallest(2.96\%), 198 records in total, and then slowly increased. The time variation of severe haze was similar to haze. Heavy haze easily occurred in January, February and march (61.16\% of the total), and less in summer (4 records, $0.61 \%$ ). Winter was the season with the most haze, and July was the month with the most haze. These results are consistent with the results of Can Zhang ${ }^{2}$, Wenhua Zhang4, which is based on the data obtained from Shapingba meteorological observatory of Chongqing. 


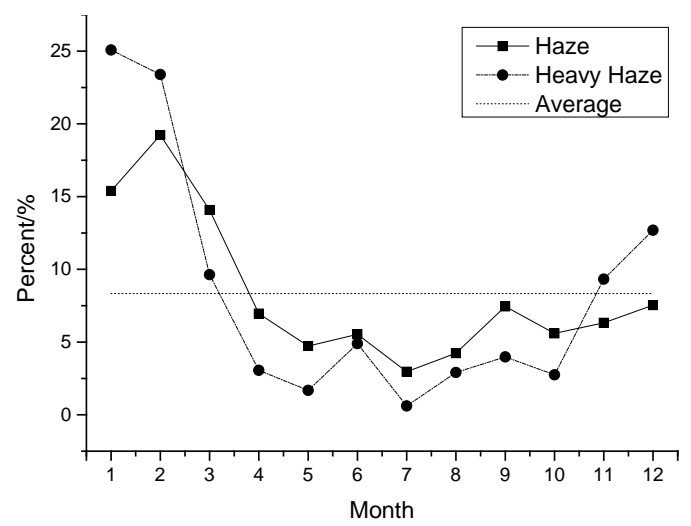

Figure 2. Monthly Frequency Distribution Graph Of Haze And Severe Haze From 2001 To 2006

As is shown in Figure 3, the frequency of haze was relatively higher between 11:00-21:00(74.54\%). The average frequency of haze in the period of these 11 hours (6.8\%) was about 3.5 times higher than the average frequency of haze in other periods (1.94\%). It gradually began to decrease and became lower than the average frequency from 21:00, and reached the lowest frequency early in the morning. (00:00 07:00). After that, it began to increase from 08:00 and became higher than the average at 11:00. The occurrence of severe haze was similar to that of the haze, but the time varied by an hour.

The change in time of haze is closely related to local relative humidity And the local humidity is affected by the solar radiation, and it has obvious changes. In colder temperatures (such as winter mornings), the relative humidity is relatively high, it is easier to have fog weather, not haze. As the temperature rises, the relative humidity decreases, the fog will turn to haze.



Figure 3. The percentage distribution of time frequency of haze and severe haze

(Beijing time; the data is smoothed by cubical smoothing algorithm with five-point approximation: the horizontal dotted line is the average frequency)

The Meteorological Conditions of Haze. Wind Direction and Wind Speed. Wind direction determines the flow of pollutants in the region, and wind speed determines the spread and concentration of pollutants in the atmosphere. Table 1 is a percentage distribution of the probability of wind direction when there is haze. It can be seen from Table 1 that When the haze occurred, it was more common to have north wind (28.67\%)and west wind(14.25\%). When the severe haze occurred, it was more likely to be north wind(24.84\%) and south wind(20.95\%). Overall, the proportion of haze and severe haze was the highest when there was north wind, with $28.67 \%$ and $24.84 \%$ respectively. Table 1 percentage distribution of the probability of wind direction when there is haze (unit: \%)

\begin{tabular}{ccccccccc}
\hline $\begin{array}{c}\text { Wind } \\
\text { direction }\end{array}$ & $\mathrm{N}$ & $\mathrm{NE}$ & $\mathrm{E}$ & $\mathrm{SE}$ & $\mathrm{S}$ & $\mathrm{SW}$ & $\mathrm{W}$ & $\mathrm{NW}$ \\
\hline $\begin{array}{c}\text { Haze } \\
\text { Severe } \\
\text { haze }\end{array}$ & 28.67 & 11.02 & 12.79 & 7.20 & 11.73 & 3.62 & 14.25 & 10.72 \\
\hline
\end{tabular}

Table 2 is the percentage distribution of wind speed probability when there is haze. It is easy to see that when the average wind speed was about $1 \sim 2 \mathrm{~m} \mathrm{~s}^{-1}$, the frequency of haze and severe haze was 
the highest, with $88.37 \%$ and $98.20 \%$ of the total. The frequency of haze reached the highest point $(37.08 \%)$ when the average wind speed was $2 \mathrm{~m} \mathrm{~s}^{-1}$. The frequency of severe haze reached the highest point $(43.88 \%)$ when the average wind speed was $1 \mathrm{~m} \mathrm{~s}^{-1}$. The haze and severe haze accounted for only $3 \%$ and $0.39 \%$ when wind speed was above $4 \mathrm{~m} \mathrm{~s}^{-1}$. The weighted average wind speed was 1.41 $\mathrm{m} \mathrm{s}^{-1}$ when severe haze occurred, which was lower than that $\left(1.95 \mathrm{~m} \mathrm{~s}^{-1}\right)$ of haze. The result of the wind speed is roughly the same as that of Wenhua Jiang ${ }^{4}$, which is based on a 14-hour observation record. Moreover, the results of this paper are based on hourly observed records, which are statistically significant.

When the wind speed is weak, the atmospheric diffusion capacity is weak, which is not conducive to the transportation of haze source. When the wind speed is high, the atmosphere is unstable, which is not conducive to the formation of inversion layer. It will lead to the spread of haze, which is not conducive to the formation of haze.

Table 2 percentage distribution of the probability of wind speed when there is haze (unit: \%)

\begin{tabular}{ccccccc}
\hline Wind speed $\mathrm{m}^{-1}$ & 0 & 1 & 2 & 3 & 4 & $>4$ \\
\hline Haze & 3.11 & 36.05 & 37.08 & 15.24 & 5.52 & 3.00 \\
Severe haze & 4.59 & 43.88 & 31.96 & 14.37 & 3.06 & 0.39 \\
\hline
\end{tabular}

Relative Humidity. The frequency percentage distribution of the relative humidity when haze occurs is shown in Table 4. As can be seen from Figure 4, the relative humidity of $50 \%-70 \%$ was the most prone to haze and severe haze range ( $88.22 \%$ and $94.50 \%$ respectively). Relative humidity of $60 \%$ was the most likely to be haze and severe haze. The weighted average relative humidity of haze and severe haze was $61.49 \%$ and $64.48 \%$ respectively. The frequency percentage was only $10.23 \%$ and $2.45 \%$ when the relative humidity was below $50 \%$. These results are basically consistent with Wenhua Jiang's ${ }^{4}$, but relative humidity will have some changes in a day.

It is easy to form fog when the air near the ground reaches or near saturation (relative humidity reaches or near 100\%). When relative humidity is between 80 and 95 percent, haze and fog can co-exist to form a mixture of fog and haze ${ }^{3}$. When relative humidity reduces further, the fog can turn into haze. Haze is also made up of wet aerosol particles, which is hard to form when relative humidity is too low (less than 40\%).

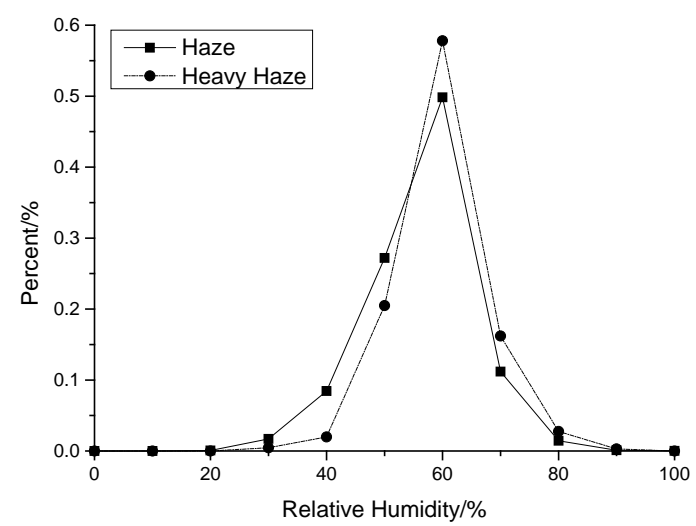

Figure 4 The frequency percentage distribution of the relative humidity when haze occurs

Concomitant Weather. The concomitant weather when haze occurs is shown in Table 3. When haze or severe haze occurred, there was no other weather conditions generally $(91.70 \%$ and $80.15 \%$ respectively), but the concomitant weather of light fog took a high proportion( $7.10 \%$ and $17.59 \%$ respectively).

Haze is usually formed when the atmosphere is relatively stable and relative humidity is mild (50-70\%). So there is usually no fog, drizzle, rain or thunderstorm. When relative humidity is high, haze and fog can co-exist. Thus, the occurrence of haze is usually with fog, and light fog mainly. When relative humidity reduces and fog evaporates, haze finally forms. 
Table 3 Statistics of concomitant weather when haze occurs (unit: \%)

\begin{tabular}{ccccccc}
\hline weather & $\begin{array}{c}\text { No other } \\
\text { weather }\end{array}$ & Light fog & Fog & Drizzle & Rain & $\begin{array}{c}\text { Thunderstor } \\
\mathrm{m}\end{array}$ \\
\hline Haze & $91.70 \%$ & $7.10 \%$ & $0.04 \%$ & $0.04 \%$ & $1.01 \%$ & $0.10 \%$ \\
Severe haze & $80.15 \%$ & $17.59 \%$ & $0.45 \%$ & $0.00 \%$ & $1.65 \%$ & $0.15 \%$ \\
\hline
\end{tabular}

\section{Conclusions}

By using hourly surface observation data in Chongqing Jiangbei Airport, this paper does elaborate analysis on characteristics of inter-annual variation, time variation and meteorological conditions. Here are the conclusions:

Through the analysis of routine observation data of Chongqing Jiangbei Airport, it is found that the characteristics such as inter-annual variability of haze, wind speed, relative humidity and so on are consistent with the analysis results based on conventional meteorological observation data of meteorological station. And the observation frequency of the airport is even higher, the observation projects are also more. The results can serve as a useful complement to conventional meteorological observation.

The haze and severe haze in Chongqing are most likely to occur in winter, with the lowest frequency of occurrence in July. The haze is relatively frequent at 11:00-21:00 and has the lowest frequency in the early morning hours (00:00 07:00).

Haze and severe haze have the highest proportion when there is north wind. The frequency of haze and severe haze is the highest when the average wind speed is in $1 \sim 2 \mathrm{~m} \mathrm{~s}^{-1}$. The frequency is lower when the wind speed is faster than $4 \mathrm{~m} \mathrm{~s}^{-1}$.

The relative humidity of $50 \%-70 \%$ is the most prone range to haze and severe haze. When haze and severe haze occur, there is usually no other weather condition, but there is a higher proportion of light fog.

\section{Acknowledgement}

Supported by "National Natural Science Foundation of China", NO. 61671237.

\section{References}

[1].China Meteorological Administration. Haze Observation and Forecast Level QX/T113-2010 [S].Beijing: Meteorological Press, 2010.

[2].Can Zhang, Zhi'en Zhou, Chongzhi Zhai,etc. Dust-Haze Weather Changes and Characteristics Analysis in Downtown Chongqing [J]. China Environmental Monitoring, 2014,30(4).69-75.

[3].Yihui Ding, Yanju Liu. The Long-Term Change Characteristcs of China's Fog and Haze and Its Relationship with Atmospheric Humidity in the Last Fifty Years [J]. China Science: Earth Science, 2014(1):37-48.

[4].Weihua Jiang, De Liu, Yonghang Chen, etc. The Characteristics of Changes in Haze Days Of Chongqing from 1980 to 2012 [J]. Arid Meteorology, 2015,33(4):602-606.

[5].Wei Huang, Chongzhi Zhai, Jiayan Yu, etc. A Preliminary Investigation of Atmospheric Pollution in Chongqing's Regional Dust-Haze [J]. Journal of Southwest Normal University (Natural Science Edition), 2012,37(7):142-146.

[6].Wenhua jiang, De Liu, Daojin Chen. Analysis of Haze Weather Characteristics and Meteorological Factors in Downtown Chongqing [J]. Journal of Southwest Normal University (Natural Science Edition), 2016, 41(5):134-138.

[7].Dui Wu. The Differences between Fog and Haze in Cities[J].Meteorology, 2006,32(4):9-15. 
[8].China Civil Aviation Administration Office of Air Traffic Management. 2007. Civil Aviation Meteorological Surface Observation Standard [Z].

[9].China Civil Aviation Administration. 2007. Standards of the Civil Aviation Industry of the People's Republic of China MH/T 4016-2007 [S].

[10].Panmao Zhai, Qinfang Zhou. 1997. Study on Climate Change of Atmospheric Water in China [J]. Journal of Applied Meteorological Science, 8 (3): 341 - 351.

[11].Cortinas J R, Bemstein B C. Robbins C C. et a1. An Analysis of Freezing Rain, Freezing Drizzle, and Ice Pellets across the United States and Canada: 1976-90[J]. Wea. Forecasting, 2004,19:377-390

[12].Jie Li, Xueliang Guo, Xiaoning Zhou, etc. The Characteristics Analysis of Freezing Rain, Freezing Drizzle and Freezing Fog from 2011 to 2013 [J]. Meteorological Science, 39 (5): 1038-1048. 